\title{
Canard cycles with three breaking mechanisms.
}

\author{
M. Caubergh and R. Roussarie
}

May 12, 2015

\begin{abstract}
This article deals with relaxation oscillations from a generic balanced canard cycle $\Gamma$ subject to three breaking parameters of Hopf or jump type. We prove that in a rescaled layer of $\Gamma$ there bifurcate at most 5 relaxation oscillations.
\end{abstract}

\section{Introduction}

We consider slow-fast systems of the form

$$
X_{\lambda, \varepsilon}:\left\{\begin{array}{l}
\dot{x}=f(x, y, \lambda, \varepsilon) \\
\dot{y}=\varepsilon g(x, y, \lambda, \varepsilon),
\end{array}\right.
$$

where $f, g$ are smooth functions. In the study of relaxation oscillations we follow the general framework as introduced in $[2,4]$.

Each canard cycle is associated with one or more breaking mechanisms. As in [5] we consider only canard cycles with $n$ generic breaking mechanisms, that may be Hopf breaking mechanisms and jump breaking mechanisms. Each mechanism depends on a so-called breaking parameter, in fact a function $a(\lambda)$ of the parameter $\lambda$. The assumed genericity is that the map $\lambda \rightarrow\left(a_{1}(\lambda), \ldots, a_{n}(\lambda)\right)$ is a local diffeomorphism. Then, we will suppose that $\lambda=a=\left(a_{1}, \ldots, a_{n}\right)$. The canard cycle exists when $a=0 \in \mathbb{R}^{n}$ and we want to study the system for $a \sim 0 \in \mathbb{R}^{n}$. A canard cycle with $n$ breaking mechanisms is associated with $n$ (horizontal fast) layers. For this reason we call such a canard cycle, indifferently: $n$-multi-layer canard cycle or canard cycle with $n$ breaking mechanisms.

Canard cycles with one breaking mechanism were largely investigated and a general result in finite codimension was obtained in [4]. Canard cycles with two breaking mechanisms were introduced in [3] and their study was completed in [7]. Canard cycles with an arbitrarily large number $n$ of mechanisms were introduced in [5]. There it is shown that bounding the limit cycles bifurcating from a generic balanced canard cycle $\Gamma$ with $n$ canard mechanisms in a rescaled layer is reduced to investigate the fixed points of a composition of translated power functions of the form

$$
\phi_{\alpha}^{\mathbf{r}}(\xi)=\phi_{\alpha_{n}}^{r_{n}} \circ \phi_{\alpha_{n-1}}^{r_{n-1}} \circ \ldots \circ \phi_{\alpha_{1}}^{r_{1}}(\xi),
$$


where $\mathbf{r}=\left(r_{1}, \ldots, r_{n}\right)$, with $r_{i} \in \mathbb{R} \backslash\{0\}$ defined in terms of divergence quantities and $\alpha=\left(\alpha_{1}, \ldots, \alpha_{n}\right)$, with $\alpha_{i} \in \mathbb{R}$ obtained by rescaling $a_{i}$. The $\phi_{\alpha_{i}}^{r_{i}}$ in (2) are translated power functions given by:

$$
\phi_{\alpha_{i}}^{r_{i}}(\xi)=\alpha_{i}+\xi^{r_{i}}, i=1, \ldots, n .
$$

The maximal number of limit cycles bifurcating from $\Gamma$, i.e its cyclicity, in a rescaled layer, is equal to the maximal number of fixed points of $\phi_{\alpha}^{\mathbf{r}}(\xi)$. For $n=1$ respectively $n=2$, the cyclicity is equal to 2 respectively 3 . This is the bound expected for an elementary catastrophe (fold resp. cusp catastrophes), although the catastrophe theory does not apply here. For $n=3$ an example by Panazzolo exhibits on generic sections in parameter space a bifurcation with 3 cusp points (see Figure 1; this example was reported in [5]).

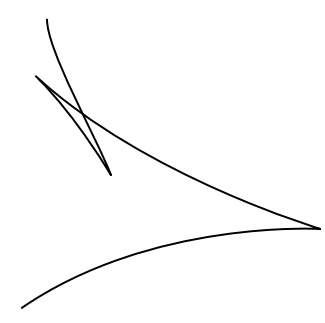

Figure 1: A planar section of the bifurcation diagram of a 3-layer canard cycle; in the bounded region 5 limit cycles are found.

In this example one finds values of the parameter with 5 fixed points for $\phi_{\alpha}^{\mathbf{r}}(\xi)$. Therefore, although this family of maps depends on a mere three dimensional parameter, its bifurcation diagram does not globally reduce to a unique elementary catastrophe. On the other hand, the cyclicity of $\Gamma$ was not obtained for $n=3$ in [5]. We obtain such a bound in this paper:

Theorem 1. Let $\Gamma$ be a balanced canard cycle with 3 breaking mechanisms, verifying the generic condition $(G)$. Then there bifurcate at most 5 limit cycles in any rescaled layer of $\Gamma$.

What is a balanced canard cycle $\Gamma$ is explained in Definition 4, in terms of the slow divergence integrals (4) associated to this canard cycle. The generic condition $(G)$ needed in Theorem 1 is specified below in (11) in terms of the divergence quantities (6). What is a rescaled layer is explained in Definition 5. Such a layer is a neighborhood of order $\varepsilon$ in the layer variables. These layer variables are used to parameterize the canard cycles near $\Gamma$ as well as the bifurcating limit cycles. Clearly, a rescaled layer does not cover a whole neighborhood of $\Gamma$; in Section 4 we further discuss this restriction.

Taking into account the Panazzolo's example, we see that the bound obtained in Theorem 1 is optimal. During the preparation of this paper, Panazzolo communicates us an article in preparation [8], where he announces that the 
equation (2), for $n=3$, has at most 5 roots. This implies of course Theorem 1 . Nevertheless, the method which is used in our paper seems to be more simple. The method of [8], using the Khovanskii theory of fewnomials [6], allows to obtain for an arbitrary $n$ the following bound:

$$
M_{n}=2^{n(2 n-1)}(n+1)^{2 n}
$$

Notice that this general formula does not give the accurate bound $M_{3}=5$, that is obtained in [8] by a direct study.

\section{General setting}

Here we recall briefly the general setting for slow fast systems and canard cycles with an arbitrary number $n$ of breaking mechanisms (see [5]).

\subsection{Some basic definitions}

The following assumptions are made on (1):

$$
\frac{\partial f}{\partial y}(x, y, \lambda, 0) \neq 0, \forall(x, y, \lambda)
$$

and

$$
\text { if } f(x, y, \lambda, 0)=\frac{\partial f}{\partial x}(x, y, \lambda, 0)=0 \text {, then } \frac{\partial^{2} f}{\partial x^{2}}(x, y, \lambda, 0) \neq 0 .
$$

For $\varepsilon=0$ we obtain the layer equation $X_{\lambda, 0}$. The set $L_{\lambda}=\{f(x, y, \lambda, 0)=0\}$ is referred to as the slow curve (of the layer equation). By the assumptions above it follows that the slow curve is a regular curve. Contact points are points where the slow curve is tangent to the horizontal direction. Let $C_{\lambda}$ be the set of these contact points. The set $L_{\lambda} \backslash C_{\lambda}$ is the union of normally hyperbolic arcs which may be of attracting type or of repelling type. Limit periodic sets appearing for $\varepsilon \rightarrow 0$ and not reduced to a singular point, are called slow fast cycles (as they are the union of slow arcs on $L_{\lambda}$ and fast orbits). They are compact invariant sets of $X_{\lambda, 0}$. Periodic orbits bifurcating from these slow fast cycles are called relaxation oscillations. A distinction is made between canard and common relaxation oscillations. The one we are interested in are the canard relaxation oscillations, which bifurcate from a slow fast cycle containing attracting as well as repelling slow arcs. Such a slow fast cycle is called canard cycle.

\subsection{Multi-layer canard cycles}

A multi-layer canard cycle is a canard cycle on which operate simultaneously $n$ breaking parameters, for some number $n \geq 1$. We suppose that the slow dynamics has no zeros on the slow arcs contained in $\Gamma$.

We have two different types of breaking mechanism: 
1. The Hopf mechanism, occurring at degenerate contact point $\left(x_{0}, y_{0}\right)$ where $g\left(x_{0}, y_{0}, 0,0\right)=0$ but $\frac{\partial g}{\partial x}\left(x_{0}, y_{0}, 0,0\right) \neq 0$. The breaking parameter is the displacement of this root of $g$.

2. The jump mechanism where a fast orbit contained in $\Gamma$ jumps from a nondegenerate contact point to another one. The breaking parameter is the vertical distance between the two contact points after perturbation.

More details about these two mechanisms can be found in [5].
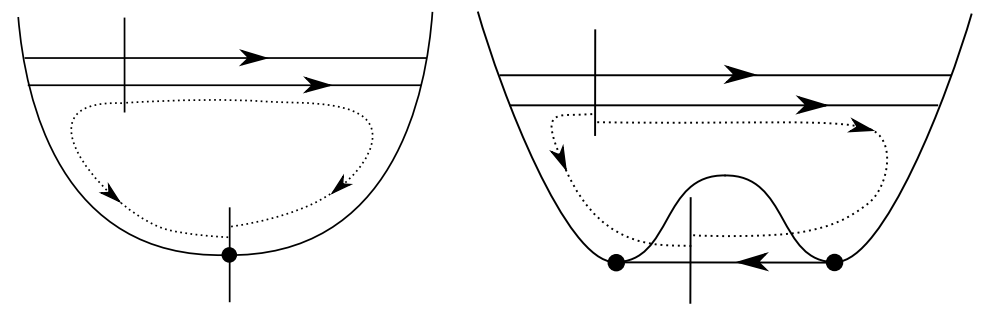

Figure 2: Canards with 1 breaking parameter; Hopf breaking mechanism on the left, jump breaking mechanism on the right.

Now, let $\mathcal{T}_{1}, \cdots, \mathcal{T}_{n}$ be the $n$ breaking mechanisms which are labeled in the order compatible with the orientation of $\Gamma$ (each $\mathcal{T}_{i}$ is situated either at a degenerate contact point or at a fast orbit between two jump points). To each $\mathcal{T}_{i}$ is associated a breaking function $a_{i}(\lambda), i=1, \ldots, n$. We suppose the generic condition:

The map $\lambda \rightarrow\left(a_{1}(\lambda), \cdots, a_{n}(\lambda)\right)$ is a local diffeomorphism at $\lambda=\lambda_{0}$.

From now on we will assume that $\lambda=\left(a_{1}, \ldots, a_{n}\right)$.

The orientation of $\Gamma$ induces a cyclic order on the breaking mechanisms and related loci; we denote them: $\mathcal{T}_{1}, \cdots, \mathcal{T}_{i}, \cdots, \mathcal{T}_{n}$, where $i$ is a cyclic index which belongs to $\mathbb{Z} / n \mathbb{Z}$.

In between two breaking mechanisms we suppose to have exactly one fast orbit (in the positive direction) having both as $\alpha$-limit and as $\omega$-limit a point in $L_{0} \backslash C_{0}$. Of course such a fast orbit has to belong to a 1-parameter family of fast orbits having both as $\alpha$-limit and as $\omega$-limit a point in $L_{0}-C_{0}$; we can call it a layer of fast orbits or fast layer.

In between a fast layer and a breaking mechanism we admit that $\Gamma$ consists of a union of attracting slow curves and fast orbits, called attracting sequence.

A fast orbit in an attracting sequence necessarily has as $\alpha$-limit a (jump) point in $C_{0}$, while we require that the $\omega$-limit be situated in $L_{0} \backslash C_{0}$.

We also require the same on $\Gamma$ when we reverse time, implying similar conditions on a succession of repelling slow arcs, as we have on a succession of attracting slow arcs. The related succession of repelling slow curves and intermediate fast orbits will be called a repelling sequence.

We return now to the layer orbits. As described before, one has a unique layer orbit $l_{i}$ in $\Gamma$, for each $i \in \mathbb{Z} / n \mathbb{Z}$. This layer orbit links the repelling sequence 
$R_{i}$ to the attracting sequence $A_{i+1}$. As already observed, each $l_{i}$ belongs to a 1-parameter family of such fast orbits (a fast layer), and as a consequence the canard cycle is a member of an $n$-parameter family of similar canard cycles. To make this point more precise, we consider a transverse section $\Sigma_{i}$ to $l_{i}$, transverse to the field $X_{0,0}$, for each $i \in \mathbb{Z} / n \mathbb{Z}$. Let $u_{i}$ be a smooth regular parametrization of $\Sigma_{i}$, such that $\Sigma_{i} \cap l_{i}$ corresponds to $u_{i}^{0}$. We can replace $l_{i}$ by $l_{i}\left(u_{i}\right)$, the fast orbit passing through the point $u_{i} \in \Sigma_{i}\left(l_{i}=l_{i}\left(u_{i}^{0}\right)\right)$. So, we have an $n$-parameter family of canard cycles $\Gamma_{u}$, parameterized by $u=\left(u_{1}, \cdots, u_{n}\right) \in \prod_{i} \Sigma_{i}$. The canard $\Gamma_{u}$ is the one containing the fast layer orbits $l_{i}\left(u_{i}\right)$, for $i \in \mathbb{Z} / n \mathbb{Z}$. To emphasize the dependence on $u_{i}$, we will write $n_{i}\left(u_{i}\right), m_{i}\left(u_{i}\right)$ for the end points of the layer orbit $l_{i}\left(u_{i}\right)$, and also $A_{i}\left(u_{i-1}\right), R_{i}\left(u_{i}\right)$ for the attracting and repelling sequences associated to the transition $\mathcal{T}_{i}$. We can assume that our canard cycle $\Gamma$ is just $\Gamma_{u^{0}}$ with $u^{0}=\left(u_{1}^{0}, \ldots, u_{n}^{0}\right)$. Parameters $u_{i}$ are called the layer variables.

\subsection{Equation of bifurcating limit cycles}

Let us consider an open connected arc $\sigma \subset L_{0} \backslash C_{0}$. Along such an arc one can consider the slow divergence integral Int $(\sigma)$, as defined in [1] for instance. For the system (1) and for an arc $\sigma$, above an interval $\left[x_{1}, x_{2}\right]$ without zero of $g$ nor contact point in its interior, we have that

$$
\operatorname{Int}(\sigma)=\operatorname{Int}\left(x_{1}, x_{2}\right)=-\int_{x_{1}}^{x_{2}} \frac{1}{g(x, y(x), 0,0)}\left(\frac{\partial f}{\partial x}(x, y(x), 0,0)\right)^{2} d x
$$

where $y(x)$ is the implicit function defined by $f(x, y(x), 0,0)=0$ along $\sigma$. The end points $x_{1}$ and $x_{2}$ may be contact points.

Let us consider now the $2 n$ integrals $I_{i, j}\left(u_{j}\right)$, defined for $i \in \mathbb{Z} / n \mathbb{Z}, \quad j=$ $i, i-1$ :

$$
I_{i, i-1}\left(u_{i-1}\right)=\operatorname{Int}\left(\sigma\left(A_{i}\left(u_{i-1}\right)\right), \quad I_{i, i}\left(u_{i}\right)=-\operatorname{Int}\left(\sigma\left(R_{i}\left(u_{i}\right)\right)\right.\right.
$$

where $\sigma\left(A_{i}\left(u_{i-1}\right)\right)$ is the union of the slow arcs which constitute the attracting sequence $A_{i}\left(u_{i-1}\right)$ and $\sigma\left(R_{i}\left(u_{i}\right)\right)$ is the union of the slow arcs which constitute the repelling sequence $R_{i}\left(u_{i}\right)$.

Remark 2. For each breaking mechanism $\mathcal{T}_{i}$ we choose one section $T_{i}$. For the Hopf mechanisms, we have to introduce as breaking parameter a rescaled parameter: $\bar{a}_{i}=\varepsilon^{-\delta} a_{i}$ for some $\delta>0$, in consequence of the blow-up needed at this point. To keep the notations homogeneous, we will also write $\bar{a}_{i}$ for the breaking parameter at a jump breaking mechanism (i.e. we write $a_{i}=\bar{a}_{i}$ for a jump breaking parameter). We globally write : $\bar{a}=\left(\bar{a}_{1}, \cdots, \bar{a}_{n}\right)$ (See [5] for more details).

We recall now an important definition:

Definition 3. We say that a function $f(z, \varepsilon)$, with $z \in \mathbb{R}^{p}$ for some $p$, is $\varepsilon$ regularly smooth in $z$ (or $\varepsilon$-regularly $\mathcal{C}^{\infty}$ in $z$ ) if $f$ is continuous and all partial derivatives of $f$ with respect to $z$ exist and are continuous in $(z, \varepsilon)$. 
We want to recall now from [5] expressions for the transition maps for $\varepsilon>0$, from the section $\Sigma_{i-1}$ to the section $T_{i}$, along the flow of $X_{a, \varepsilon}$, and from $\Sigma_{i}$ to $T_{i}$ along the flow of $-X_{a, \varepsilon}$ (reversing time). There exist functions $\tilde{I}_{i, j}\left(u_{j}, \bar{a}, \varepsilon\right)$ which are $\varepsilon$-regularly $\mathcal{C}^{\infty}$ in $\left(u_{j}, \bar{a}\right)$, such that

$$
\tilde{I}_{i, j}\left(u_{j}, 0,0\right)=I_{i, j}\left(u_{j}\right) \text { for } i \in \mathbb{Z} / n \mathbb{Z}, \quad j=i-1, i
$$

and such that the transition maps have the following expressions:

1. From $\Sigma_{i-1}$ to $T_{i}: u_{i-1} \rightarrow \exp \frac{\tilde{I}_{i, i-1}\left(u_{i-1}, \bar{a}, \varepsilon\right)}{\varepsilon}+f_{i, i-1}(\bar{a}, \varepsilon)$,

2. From $\Sigma_{i}$ to $T_{i}: u_{i} \rightarrow \exp \frac{\tilde{I}_{i, i}\left(u_{i}, \bar{a}, \varepsilon\right)}{\varepsilon}+f_{i, i}(\bar{a}, \varepsilon)$,

with $f_{i, j}$ functions that are $\varepsilon$-regularly smooth in $\bar{a}$, and $\frac{\partial f_{i, j}}{\partial \bar{a}_{i}}(0,0)>0$. One deduces in [5] the following system of $n$ equations for the limit cycles:

$$
\exp \frac{\tilde{I}_{i, i-1}\left(u_{i-1}, \bar{a}, \varepsilon\right)}{\varepsilon}-\exp \frac{\tilde{I}_{i, i}\left(u_{i}, \bar{a}, \varepsilon\right)}{\varepsilon}=\bar{a}_{i} \text { for } i=1, \cdots, n
$$

with new functions $\tilde{I}_{i, j}$ which differ from the previous ones by terms of order $O(\varepsilon)$, which are $\varepsilon$-regularly $\mathcal{C}^{\infty}$ in $(u, \bar{a})$.

In Figures 2 and 3 the transition maps are indicated by dotted lines.

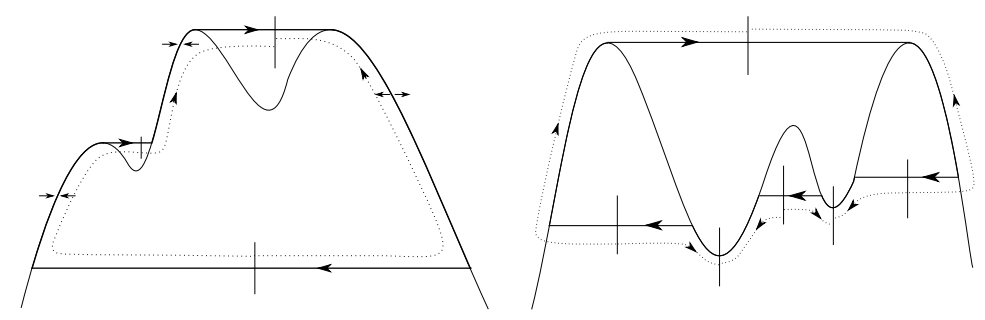

Figure 3: On the left a canard cycle with 1 breaking mechanism (jump) and exhibiting an attracting sequence. On the right a canard cycle with 3 breaking mechanisms (1 jump and 2 Hopf).

\subsection{Rescaling generic balanced canard cycles}

Recall $\Gamma$ is the canard cycle $\Gamma_{u}$ associated to $u=u^{0}$.

Definition 4. The canard cycle $\Gamma$ is said to be balanced if the integrals $I_{i, j}$ verify the following conditions

$$
I_{i, i}\left(u_{i}^{0}\right)=I_{i, i-1}\left(u_{i-1}^{0}\right) \text { for } i \in \mathbb{Z} / n \mathbb{Z} .
$$


Let us suppose that $\Gamma$ is a balanced canard cycle. Then $\Gamma$ is said to be generic if it verifies the generic condition

$$
(G): \prod_{i=1}^{n} I_{i, i}^{\prime}\left(u_{i}^{0}\right) \neq \prod_{i=1}^{n} I_{i, i-1}^{\prime}\left(u_{i-1}^{0}\right)
$$

We assume from now on that $\Gamma$ is a generic balanced canard cycle. It is proven in $[5]$ that there exists an $\varepsilon$-regularly function $u_{i}(\bar{a}, \varepsilon)$ such that

$$
\tilde{I}_{i, i}\left(u_{i}(\bar{a}, \varepsilon), \bar{a}, \varepsilon\right)=\tilde{I}_{i, i-1}\left(u_{i-1}(\bar{a}, \varepsilon), \bar{a}, \varepsilon\right),
$$

for all $\varepsilon>0$, small enough. We write $u(\bar{a}, \varepsilon)=\left(u_{1}(\bar{a}, \varepsilon), \ldots, u_{n}(\bar{a}, \varepsilon)\right)$.

We can introduce now the rescaled layer variables:

Definition 5. Let us suppose that $\Gamma$ is a generic balanced canard cycle and let $u(\bar{a}, \varepsilon)=\left(u_{1}(\bar{a}, \varepsilon), \ldots, u_{n}(\bar{a}, \varepsilon)\right)$ the application defined above. For each $i=$ $1, \cdots, n$, the rescaled layer variable $U_{i}$ is defined by

$$
u_{i}=u_{i}(\bar{a}, \varepsilon)+\varepsilon U_{i} .
$$

Taking $K_{i}>0$, for $i=1, \ldots, n$ arbitrarily large constants, we define a rescaled layer by taking $U_{i} \in\left[-K_{i}, K_{i}\right]$, for $i=1, \ldots, n$, and $\varepsilon$ small enough.

Introduce:

$$
I_{i}^{0}(\bar{a}, \varepsilon)=\tilde{I}_{i, i}\left(u_{i}(\bar{a}, \varepsilon), \bar{a}, \varepsilon\right)=\tilde{I}_{i, i-1}\left(u_{i-1}(\bar{a}, \varepsilon), \bar{a}, \varepsilon\right) \text { and } I_{i, j}^{1}=I_{i, j}^{\prime}\left(u_{i}^{0}\right),
$$

then we have that:

$$
\tilde{I}_{i, j}\left(u_{j}, \bar{a}, \varepsilon\right)=I_{i}^{0}(\bar{a}, \varepsilon)+\varepsilon I_{i, j}^{1} U_{j}(1+O(\varepsilon)),
$$

where $O(\varepsilon)$ is $\varepsilon$-regularly smooth in $U_{j}$.

Substituting (7) in the equations (5) we obtain, for $i=1, \ldots, n$, the rescaled equations:

$$
\exp \left(I_{i, i}^{1} U_{i}(1+O(\varepsilon))\right)-\exp \left(I_{i, i-1}^{1} U_{i-1}(1+O(\varepsilon))\right)=\alpha_{i},
$$

for rescaled parameter variables $\alpha_{i}=\bar{a}_{i} \exp \left(-I_{i}^{0}(\bar{a}, \varepsilon) / \varepsilon\right)$. To simplify the notation further, we also write: $I_{i, i}^{1}\left(u_{i}^{0}\right)=\tau_{i}, \quad I_{i, i-1}^{1}\left(u_{i-1}^{0}\right)=\nu_{i-1}$ and $r_{i}=\frac{\nu_{i-1}}{\tau_{i-1}}$ for $i \in \mathbb{Z} / n \mathbb{Z}$. At the parameter $(\alpha, r)$ one associates in [5] the translated power function:

$$
\Phi_{\alpha}^{r}(\xi)=\alpha+\xi^{r} .
$$

Now, putting $\xi=\exp U_{n}$, it is proven in [5] that the system of equations (8) reduces to a one-dimensional fixed point equation for a map: $\xi \rightarrow \varphi_{\alpha}^{r}(\xi)+O(\varepsilon)$ where

$$
\varphi_{\alpha}^{r}=\phi_{\alpha_{n}}^{r_{n}} \circ \cdots \circ \phi_{\alpha_{1}}^{r_{1}},
$$

and $O(\varepsilon)$ is $\varepsilon$-regularly smooth in $(\xi, \alpha, r)$. 


\section{System with three breaking parameters}

In this section we particularize the general setting to systems with three breaking parameter mechanisms. Each of these mechanisms may be of Hopf or jump type. Figures 3 and 4 present examples of such system.

We will denote by $u, v, w$ the layer variables, by $I(u), J(v), K(u), L(w), M(v)$ and $N(w)$ the 6 involved slow fast integrals and by $a, b, c$ the three breaking parameters. As above, we change the parameter $(a, b, c)$ for the new parameter $(\bar{a}, \bar{b}, \bar{c})$ to take into account the possibility of Hopf type mechanisms (see Remark 2). We assume that $\lambda=(\bar{a}, \bar{b}, \bar{c})$ is the whole parameter of $X_{\lambda, \varepsilon}$.

As consequence of their basic properties, the slow divergence integrals are strictly negative and with strictly non-zero derivative:

$$
I^{\prime}(u) \neq 0, K^{\prime}(u) \neq 0, J^{\prime}(v) \neq 0, M^{\prime}(v) \neq 0, L^{\prime}(w) \neq 0, N^{\prime}(w) \neq 0 .
$$

We consider the canard cycle $\Gamma=\Gamma_{\left(u^{0}, v^{0}, w^{0}\right)}$. It is supposed to be balanced, which here reads as

$$
I\left(u^{0}\right)=J\left(v^{0}\right), K\left(u^{0}\right)=L\left(w^{0}\right) \text { and } M\left(v^{0}\right)=N\left(w^{0}\right) .
$$

Definition 6. The divergence quantities are the derivatives of the 6 divergence integrals, computed along the canard cycle:

$$
\left\{\begin{array}{l}
I_{1}=I^{\prime}\left(u^{0}\right), J_{1}=J^{\prime}\left(v^{0}\right), K_{1}=K^{\prime}\left(u^{0}\right), \\
L_{1}=L^{\prime}\left(w^{0}\right), M_{1}=M^{\prime}\left(v^{0}\right), N_{1}=N^{\prime}\left(w^{0}\right) .
\end{array}\right.
$$

The canard cycle $\Gamma$ is also supposed to be generic. This means that it verifies the property $(G)$ in $(6)$, which here reads as

$$
G=\frac{I_{1} L_{1} M_{1}}{J_{1} K_{1} N_{1}} \neq 1
$$

\subsection{An example}

Consider the slow fast system in the Liénard plane

$$
\left\{\begin{array}{l}
\dot{x}=y+\frac{1}{2} x^{2}-\frac{1}{4} x^{4} \\
\dot{y}=\varepsilon g_{d \delta_{1} \delta_{2}}(x, a, b, c),
\end{array}\right.
$$

with

$$
g_{d \delta_{1} \delta_{2}}(x, a, b, c)=\frac{(x-a)(x+1-b)(x-1-c)\left(x-d-\delta_{1}\right)(x+d)}{1+\delta_{2} x} .
$$

We see that the slow curve has two minima at $x=-1$ and $x=1$ and one maximum at $x=0$. The values of the minima is equal to $-\frac{1}{4}$ and the value of the maximum is equal to 0 . The breaking parameters are $(a, b, c)$ and 


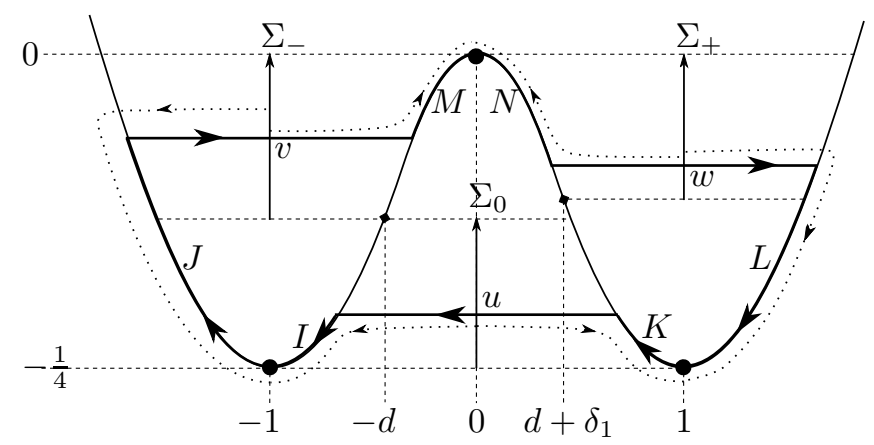

Figure 4: Canard cycle $\Gamma_{(u, v, w)}$ for (12) with 3 Hopf breaking parameters.

for $(a, b, c)=(0,0,0)$ we have three Hopf mechanisms of canard cycles. The parameters $\left(d, \delta_{1}, \delta_{2}\right)$ are constants that have to be chosen such that there exists a generic balanced canard cycle. We will not give a complete proof of this claim. We content ourselves in giving some indications which support it.

We write $F(x)=-\frac{1}{2} x^{2}+\frac{1}{4} x^{4}$. We will choose $\left.d \in\right] 0,1\left[\right.$ and $\delta_{1}, \delta_{2}$ small enough such that $\left.d+\delta_{1} \in\right] 0,1\left[\right.$ and $\left|\delta_{2}\right|<1$. There are two singularities of the slow dynamics at points $(-d, F(d))$ and $\left(d+\delta_{1}, F\left(d+\delta_{1}\right)\right)$ on the interior branches of the slow curve. The orientation of the slow dynamics is shown in Figure 4. We choose the three layer sections to be $\Sigma_{0}=\{x=0\}, \Sigma_{-1}=\{x=-1\}$ and $\Sigma_{+}=\{x=1\}$, with parametrization $u, v, w$ respectively, being equal to the coordinate $y$. We take $u \in]-\frac{1}{4}, \operatorname{Inf}\left\{F(d), F\left(d+\delta_{1}\right)\right\}[, v \in] F(d), 0[$ and $w \in] F\left(d+\delta_{1}\right), 0[$. Then, for any convenient value $(u, v, w)$ we have a canard cycle $\Gamma_{(u, v, w)}$.

We write $g_{d \delta_{1} \delta_{2}}(x)=g_{d \delta_{1} \delta_{2}}(x, 0,0,0)$. If $f(x)=\frac{\partial F}{\partial x}$, we have that

$$
g_{d \delta_{1} \delta_{2}}(x)=f(x) \frac{\left(x-d+\delta_{1}\right)(x+d)}{1+\delta_{2} x}
$$

and from (3) we obtain the following expression for the slow divergence integral:

$$
\operatorname{Int}\left(x_{1}, x_{2}\right)=\int_{x_{1}}^{x_{2}} \frac{1+\delta_{2} x}{\left(x-d+\delta_{1}\right)(x+d)} f(x) d x .
$$

For any $y \in]-\frac{1}{4}, 0\left[\right.$ we let $-x_{+}(y)<-x_{0}(y)<x_{0}(y)<x_{+}(y)$ be the four roots of the equation $\{F(x)=y\}$. The six slow divergence integrals are given by (see Figure 4):

$$
I(u)=\operatorname{Int}\left(-x_{0}(u),-1\right), K(u)=\operatorname{Int}\left(x_{0}(u), 1\right), J(v)=\operatorname{Int}\left(-x_{+}(v),-1\right)
$$

and

$$
M(v)=\operatorname{Int}\left(-x_{0}(v), 0\right), L(w)=\operatorname{Int}\left(x_{+}(w), 1\right), N(w)=\operatorname{Int}\left(x_{0}(w), 0\right) .
$$


We now explain how to find a generic balanced canard cycle. First, for $\delta_{1}=$ $\delta_{2}=0$, the system is symmetric with respect to the $O y$-axis. Then the integrals $I, K$ are identical and also the pairs $J, L$ and $M, N$. Moreover the four integrals $I, K, M, N$ vary from $-\infty$ to 0 and the two integrals $J, L$ have a bounded variation. Taking for instance any value for $v$ we have a unique value $u(v)$ such that the symmetric canard cycle $\Gamma_{u(v) v v}$ is balanced. Of course, as this canard cycle belongs to a 1-parameter family of balanced canard cycles, it cannot verify the condition $(G)$. However, it seems reasonable to think that there exist choices of the constants $\left(d, \delta_{1}, \delta_{2}\right)$ which break this symmetry and for which there exists a generic balanced canard cycle.

\subsection{System of equations for relaxation oscillations}

The system of governing equations for relaxation oscillations is given by

$$
\left\{\begin{aligned}
\exp (\tilde{I}(u, \lambda, \varepsilon) / \varepsilon)-\exp (\tilde{J}(v, \lambda, \varepsilon) / \varepsilon) & =\bar{a} \\
\exp (\tilde{K}(u, \lambda, \varepsilon) / \varepsilon) & -\exp (\tilde{L}(w, \lambda, \varepsilon) / \varepsilon) \\
\exp (\tilde{M}(v, \lambda, \varepsilon) / \varepsilon)-\exp (\tilde{N}(w, \lambda, \varepsilon) / \varepsilon) & =\bar{c}
\end{aligned}\right.
$$

where $\lambda=(\bar{a}, \bar{b}, \bar{c})$ is near $(0,0,0)$ and the solutions $(u, v, w)$ that we are looking for are near $\left(u^{0}, v^{0}, w^{0}\right)$. Since $\Gamma$ is a generic balanced canard cycle (i.e. we have (9) and (11)), there exist $\varepsilon$-regularly smooth functions $u(\lambda, \varepsilon), v(\lambda, \varepsilon), w(\lambda, \varepsilon)$, with $u(\lambda, 0)=u^{0}, v(\lambda, 0)=v^{0}$ and $w(\lambda, 0)=w^{0}$, such that:

$$
\begin{aligned}
\tilde{I}(u(\lambda, \varepsilon), \lambda, \varepsilon) & \equiv \tilde{J}(v(\lambda, \varepsilon), \lambda, \varepsilon) \\
\tilde{K}(u(\lambda, \varepsilon), \lambda, \varepsilon) & \equiv \tilde{L}(w(\lambda, \varepsilon), \lambda, \varepsilon) \\
\tilde{M}(v(\lambda, \varepsilon), \lambda, \varepsilon) & \equiv \tilde{N}(w(\lambda, \varepsilon), \lambda, \varepsilon) .
\end{aligned}
$$

We introduce the translated layer variables:

$$
\bar{u}=u-u(\lambda, \varepsilon), \bar{v}=v-v(\lambda, \varepsilon), \bar{w}=w-w(\lambda, \varepsilon),
$$

and we can expand:

$$
\tilde{I}(u, \lambda, \varepsilon)=\tilde{I}_{0}(\lambda, \varepsilon)+\tilde{I}_{1}(\lambda, \varepsilon) \bar{u}\left(1+\bar{u}^{2}\right),
$$

and also the other functions $\tilde{J}, \tilde{K}, \ldots$ Let us notice that we have

$$
\tilde{I}_{0}(\lambda, 0) \equiv I\left(u^{0}\right), \tilde{I}_{1}(\lambda, 0) \equiv I_{1}
$$

and similar $\varepsilon$-limits for the other functions $\tilde{J}, \tilde{K}, \ldots$ Next we introduce the rescaled parameter variables

$$
\alpha=\bar{a} \exp (-\tilde{I}(0, \lambda, \varepsilon) / \varepsilon), \beta=\bar{b} \exp (-\tilde{K}(0, \lambda, \varepsilon) / \varepsilon), \gamma=\bar{c} \exp (-\tilde{M}(0, \lambda, \varepsilon) / \varepsilon),
$$

and we write $\tilde{I}_{1}(\lambda, \varepsilon)=\tilde{I}^{\prime}(0, \lambda, \varepsilon), \tilde{J}_{1}(\lambda, \varepsilon)=\tilde{J}^{\prime}(0, \lambda, \varepsilon), \ldots$; to simplify reading, in the sequel we shortly write $\tilde{I}_{1}=\tilde{I}_{1}(\lambda, \varepsilon), \tilde{J}_{1}=\tilde{J}_{1}(\lambda, \varepsilon), \ldots$ although they do 
depend on $(\lambda, \varepsilon)$. Then the system of governing equations for limit cycles (15) for $(\bar{u}, \bar{v}, \bar{w})$ near $(0,0,0)$, is reduced to

$$
\left\{\begin{array}{cl}
\exp \left(\tilde{I}_{1} \bar{u}(1+O(\bar{u})) / \varepsilon\right)-\exp \left(\tilde{J}_{1} \bar{v}(1+O(\bar{v})) / \varepsilon\right) & =\alpha \\
\exp \left(\tilde{K}_{1} \bar{u}(1+O(\bar{u})) / \varepsilon\right)-\exp \left(\tilde{L}_{1} \bar{w}(1+O(\bar{v})) / \varepsilon\right) & =\beta \\
\exp \left(\tilde{M}_{1} \bar{v}(1+O(\bar{v})) / \varepsilon\right)-\exp \left(\tilde{N}_{1} \bar{w}(1+O(\bar{w})) / \varepsilon\right) & =\gamma
\end{array}\right.
$$

\subsection{Khovanskii's reduction of the system of equations}

To control the number of solutions of (17) we use a Khovanskii's method. This approach is quite similar to the first step of the method used in [7] for canard cycles with 2 breaking parameters. In the system (17) we replace one equation by an equation equivalent to $D(\bar{u}, \bar{v}, \bar{w}, \lambda, \varepsilon)=0$, where $D$ is the Jacobian determinant of the left-hand side of (17) with respect to $(\bar{u}, \bar{v}, \bar{w})$. Since we have

$\frac{\partial}{\partial \bar{u}} \exp \frac{\tilde{I}_{1} \bar{u}(1+O(\bar{u}))}{\varepsilon}=\frac{\tilde{I}_{1}(1+O(\bar{u}))}{\varepsilon} \exp \frac{\tilde{I}_{1} \bar{u}(1+O(\bar{u}))}{\varepsilon}=\frac{\tilde{I}_{1}}{\varepsilon} \exp \frac{\tilde{I}_{1} \tilde{u}(1+O(\bar{u}))}{\varepsilon}$,

and analogous expressions for the other derivatives, we obtain that

$$
\begin{aligned}
D= & \frac{\tilde{I}_{1} \tilde{L}_{1} \tilde{M}_{1}}{\varepsilon^{3}} \exp \left[\frac{\tilde{I}_{1} \bar{u}(1+O(\bar{u}))+\tilde{M}_{1} \bar{v}(1+O(\bar{v}))+\tilde{L}_{1} \bar{w}(1+O(\bar{w}))}{\varepsilon}\right] \\
& -\frac{\tilde{J}_{1} \tilde{K}_{1} \tilde{N}_{1}}{\varepsilon^{3}} \exp \left[\frac{\tilde{K}_{1} \bar{u}(1+O(\bar{u}))+\tilde{J}_{1} \bar{v}(1+O(\bar{v}))+\tilde{N}_{1} \bar{w}(1+O(\bar{w}))}{\varepsilon}\right] .
\end{aligned}
$$

We write $\tilde{G}=\frac{\tilde{I}_{1} \tilde{L}_{1} \tilde{M}_{1}}{\tilde{J}_{1} \tilde{K}_{1} \tilde{N}_{1}}=G+O(\varepsilon)$, with a term $O(\varepsilon)$ which is $\varepsilon$-regularly smooth in $\lambda$ and with $G$ as defined in (11). If $G<0$, the Jacobian determinant is locally non-zero. Then, the system (17) has locally at most one solution. From now on we will suppose that $G>0$. In this case, the equation $D=0$ is equivalent to

$$
\left(\tilde{I}_{1}-\tilde{K}_{1}\right) \bar{u}+\left(\tilde{M}_{1}-\tilde{J}_{1}\right) \bar{v}+\left(\tilde{L}_{1}-\tilde{N}_{1}\right) \bar{w}+\varepsilon \ln \tilde{G}+O\left(\|(\bar{u}, \bar{v}, \bar{w})\|^{2}\right)=0 .
$$

Under the generic condition $(\mathrm{G})$, as given in (11), at least one of the three coefficients $I_{1}-K_{1}, J_{1}-M_{1}$ or $L_{1}-N_{1}$ is different from 0 . Without loss of generality, we can assume that $L_{1}-N_{1} \neq 0$. Then, using Implicit Function Theorem, (18) can be solved for $\bar{w}$. We thus obtain a function $\bar{w}$, that is $\varepsilon$ regularly smooth in $(\bar{u}, \bar{v}, \lambda)$ and from (18) we find:

$$
\bar{w}(\bar{u}, \bar{v}, \lambda, \varepsilon)=-\frac{\tilde{I}_{1}-\tilde{K}_{1}}{\tilde{L}_{1}-\tilde{N}_{1}} \bar{u}-\frac{\tilde{M}_{1}-\tilde{J}_{1}}{\tilde{L}_{1}-\tilde{N}_{1}} \bar{v}-\varepsilon \ln \tilde{G}+O\left(\|(\bar{u}, \bar{v})\|^{2}\right),
$$

In order to simplify the system of equations, we consider a coordinate transformation of the layer variables

$$
(\bar{u}, \bar{v}) \mapsto(\tilde{u}, \tilde{v}),
$$


where $\tilde{u}=\tilde{I}_{1} \bar{u}(1+O(\bar{u})), \tilde{v}=\tilde{J}_{1} \bar{v}(1+O(\bar{v}))$, are the arguments of the exponential functions appearing in the first equation in (17). After this change of variables, the function (19) is replaced by the function $\tilde{w}$ with

$$
\tilde{w}(\tilde{u}, \tilde{v}, \lambda, \varepsilon)=-\frac{\tilde{I}_{1}-\tilde{K}_{1}}{\tilde{L}_{1}-\tilde{N}_{1}} \frac{\tilde{u}}{\tilde{I}_{1}}-\frac{\tilde{M}_{1}-\tilde{J}_{1}}{\tilde{L}_{1}-\tilde{N}_{1}} \frac{\tilde{v}}{\tilde{J}_{1}}-\varepsilon \ln \tilde{G}+O\left(\|(\tilde{u}, \tilde{v})\|^{2}\right) .
$$

The Khovanskii's method consists in replacing one of the three equations in (17), we choose the last one, by the equation $D=0$, which for $\|(\bar{u}, \bar{v}, \bar{w})\| \rightarrow 0$ is equivalent to the equation $\bar{w}=\tilde{w}(\tilde{u}, \tilde{v}, \lambda, \varepsilon)$. In this way, we can eliminate $\bar{w}$ in the two first equations of (17) to obtain the following system of two equations in $(\tilde{u}, \tilde{v})$

$$
\left\{\begin{array}{cl}
\exp \frac{\tilde{u}}{\varepsilon}-\exp \frac{\tilde{v}}{\varepsilon} & =\alpha, \\
\exp \frac{\tilde{\sigma} \tilde{u}(1+O(\tilde{u}))}{\varepsilon}-\exp \frac{\tilde{\sigma}_{1} \tilde{u}+\tilde{\sigma}_{2} \tilde{v}-\varepsilon \ln \tilde{G}+O\left(\|(\tilde{u}, \tilde{v})\|^{2}\right)}{\varepsilon} & =\beta,
\end{array}\right.
$$

where $\tilde{\sigma}, \tilde{\sigma}_{1}, \tilde{\sigma}_{2}$ are $\varepsilon$-regularly functions in $\lambda$ given by

$$
\tilde{\sigma}=\frac{\tilde{K}_{1}}{\tilde{I}_{1}}, \tilde{\sigma}_{1}=-\frac{\tilde{L}_{1}\left(\tilde{I}_{1}-\tilde{K}_{1}\right)}{\tilde{I}_{1}\left(\tilde{L}_{1}-\tilde{N}_{1}\right)}, \tilde{\sigma}_{2}=-\frac{\tilde{L}_{1}\left(\tilde{M}_{1}-\tilde{J}_{1}\right)}{\tilde{J}_{1}\left(\tilde{L}_{1}-\tilde{N}_{1}\right)} .
$$

The system of equations (20) counts the number of contact points between the foliation defined by the last equation in (17) and the curves defined by the two first equations of (17). Therefore, for a given value of the parameter $(\lambda, \varepsilon)$, the maximal number of solutions $(u, v, w)$ of $(17)$ is bounded by $1+$ the number of solutions $(\tilde{u}, \tilde{v})$ of $(20)$.

Notice that system (20) is very similar to the one encountered in [7] for the case of two breaking parameters. The only difference is that the second term in the second equation of (20) depends on two variables $\tilde{u}$ and $\tilde{v}$ and not just on the single variable $\tilde{v}$. This simple fact prevents us to proceed to further steps of Khovanskii's method as it was possible in [7]. For this reason we now have to restrict the study to a rescaled layer.

\subsection{Rescaled system of equations}

As we announced in Section 1, the rescaling of equation (17) reduces the question of bounding the number of limit cycles bifurcating in a rescaled layer of $\Gamma$ to the question of the number of fixed points for a fewnomial type map, here the composition of three translation power functions:

$$
\xi \mapsto-\alpha+\left(\beta+\left(-\gamma+\xi^{r_{1}}\right)^{r_{2}}\right)^{r_{3}},
$$

where $r_{1}=\frac{M_{1}}{J_{1}}, r_{2}=\frac{L_{1}}{N_{1}}, r_{3}=\frac{I_{1}}{K_{1}}$. As we commented in Section 1 a direct approach of this question is announced in [8], to obtain 5 as bound. In the present paper we will obtain this bound by rescaling system (20). As this system 
is much simpler than (17), we believe that our proof is also much simpler than a direct study of (21).

We now enter in the proof of Theorem 1 . To this end, we rescale the variables $\tilde{u}, \tilde{v}$ by

$$
\tilde{u}=\varepsilon U, \quad \tilde{v}=\varepsilon V,
$$

with $U, V$ in arbitrarily large compact intervals. Next, we make the change of variables

$$
\xi=\exp U, \eta=\exp V
$$

where now $\xi, \eta$ are to be considered in arbitrarily compact intervals in $] 0,+\infty[$. Recalling the notation of the divergence quantities in (10) and (16) we write

$$
\sigma=\frac{K_{1}}{I_{1}}, \sigma_{1}=-\frac{L_{1}\left(I_{1}-K_{1}\right)}{I_{1}\left(L_{1}-N_{1}\right)}, \sigma_{2}=-\frac{L_{1}\left(M_{1}-J_{1}\right)}{J_{1}\left(L_{1}-N_{1}\right)}
$$

then system (20) reads as

$$
\left\{\begin{array}{l}
\xi-\eta=\alpha \\
\xi^{\sigma}-G^{-1} \xi^{\sigma_{1}} \eta^{\sigma_{2}}+O(\varepsilon)=\beta,
\end{array}\right.
$$

where the uniformity of the term $O(\varepsilon)$ is relative to the choice of the compact domain for $(\xi, \eta, \alpha, \beta)$. Moreover this term is $\varepsilon$-regularly smooth in $(\xi, \eta, \alpha, \beta)$. Hence, by substitution of $\eta=\xi-\alpha$ in the second equation we obtain a onedimensional equation:

$$
\xi^{\sigma}-G^{-1} \xi^{\sigma_{1}}(\xi-\alpha)^{\sigma_{2}}-\beta+O(\varepsilon)=0,
$$

where again the term $O(\varepsilon)$ is uniform with respect to the choice of the compact domain for $(\xi, \alpha, \beta)$ and it is $\varepsilon$-regularly smooth in $(\xi, \alpha, \beta)$.

Theorem 1 follows from next claim:

Proposition 7. For any fixed $\left(\sigma, \sigma_{1}, \sigma_{2}\right)$ and $(\alpha, \beta) \in \mathbb{R}^{2}$, the fewnomial type function

$$
\varphi_{\sigma, \sigma_{1}, \sigma_{2}}(\xi, \alpha, \beta)=\xi^{\sigma}-G^{-1} \xi^{\sigma_{1}}(\xi-\alpha)^{\sigma_{2}}-\beta,
$$

has at most 4 roots in $\xi$ counted with their multiplicities in $] \alpha, \infty[\cap] 0, \infty[$.

Before proving Proposition 7 we first show how to deduce Theorem 1 from it. As we are looking for solutions $(\xi, \eta)$ for system $(22)$, in some compact set, we see that we can also restrict $(\alpha, \beta)$ to some compact set of $\mathbb{R}^{2}$. Now the term $O(\varepsilon)$ in $(23)$ is uniform in compact sets for $(\xi, \alpha, \beta)$ and $\varepsilon$-regularly smooth.

Let $A$ be a compact interval in $] 0,+\infty[$. As the property to have at most 4 roots in $\xi$ counted with their multiplicities is stable under smooth perturbations on compact domains, it follows that the left-hand side of (23) is a function with less than 4 roots in $A$ for $\varepsilon$ small enough. This implies that system (22) has less than 4 solutions on a given compact domain for $\varepsilon$ small enough, from which Theorem 1 is proven. 
Proof of Proposition 7 First we consider the case $\alpha=0$. Then

$$
\varphi_{\sigma, \sigma_{1}, \sigma_{2}}(\xi, \alpha, \beta)=\xi^{\sigma}-G^{-1} \xi^{\sigma_{1}+\sigma_{2}}-\beta
$$

This function has at most two roots counted with their multiplicities if $\sigma \neq$ $\sigma_{1}+\sigma_{2}$, and, as $G \neq 1$, has at most a simple root if $\sigma=\sigma_{1}+\sigma_{2}$.

Next we suppose that $\alpha \neq 0$. To study the zeroes $\xi$ of (24) in function of $(\alpha, \beta)$, we distinguish the case $\alpha>0$ and $\alpha<0$. As $\left(\sigma, \sigma_{1}, \sigma_{2}\right)$ is fixed, we denote the function $\varphi_{\sigma, \sigma_{1}, \sigma_{2}}$ simply by $\varphi$.

1. Case $\alpha>0$. We introduce the variable $\mu$ by $\xi=\alpha(1+\mu)$ with $\mu>0$ (since $a \mu=\xi-\alpha>0$ ). Then $\varphi$ transforms into

$$
\varphi_{+}(\mu) \equiv \varphi(\alpha(1+\mu), \alpha, \beta)=\alpha^{\sigma}(1+\mu)^{\sigma}-G^{-1} \alpha^{\sigma_{1}+\sigma_{2}} \mu^{\sigma_{2}}(1+\mu)^{\sigma_{1}}-\beta .
$$

To bound the zeroes of $\varphi_{+}$we apply a division-derivation algorithm. Hence,

$\frac{\partial \varphi_{+}}{\partial \mu}(\mu)=\sigma \alpha^{\sigma}(1+\mu)^{\sigma-1}-G^{-1} \alpha^{\sigma_{1}+\sigma_{2}}\left[\sigma_{1}(1+\mu)^{\sigma_{1}-1} \mu^{\sigma_{2}}+\sigma_{2}(1+\mu)^{\sigma_{1}} \mu^{\sigma_{2}-1}\right]$

and so

$$
(1+\mu)^{1-\sigma} \frac{\partial \varphi_{+}}{\partial \mu}(\mu)=\sigma \alpha^{\sigma}-G^{-1} \alpha^{\sigma_{1}+\sigma_{2}} \varphi_{+}^{1}(\mu),
$$

where $\varphi_{+}^{1}(\mu)=(1+\mu)^{1-\sigma}\left[\sigma_{1}(1+\mu)^{\sigma_{1}-1} \mu^{\sigma_{2}}+\sigma_{2}(1+\mu)^{\sigma_{1}} \mu^{\sigma_{2}-1}\right]$. Then

$$
\begin{gathered}
\frac{\partial \varphi_{+}^{1}}{\partial \mu}(\mu)=(1+\mu)^{\sigma_{1}-\sigma-1} \mu^{\sigma_{2}-2} \varphi_{+}^{2}(\mu), \text { where } \\
\varphi_{+}^{2}(\mu)=\sigma_{2}\left(\sigma-2 \sigma_{1}-1\right)(1+\mu) \mu+\sigma_{1}\left(\sigma-\sigma_{1}\right) \mu^{2}+\sigma_{2}\left(1-\sigma_{2}\right)(1+\mu)^{2} .
\end{gathered}
$$

This last function is a polynomial of degree 2 in $\mu$, more precisely:

$$
\varphi_{+}^{2}(\mu)=\left(\sigma_{1}+\sigma_{2}\right)\left(\sigma_{1}+\sigma_{2}-\sigma\right) \mu^{2}+\sigma_{2}\left(2 \sigma_{1}+2 \sigma_{2}-\sigma-1\right) \mu+\sigma_{2}\left(\sigma_{2}-1\right) .
$$

The number of positive zeroes $\mu$ for $\varphi_{+}^{2}$ corresponds to the one for $\frac{\partial^{2} \varphi_{+}}{\partial \mu^{2}}$. A direct and easy analysis shows that this polynomial is identically to zero if and only if the triple $\left(\sigma, \sigma_{1}, \sigma_{2}\right)$ is equal to $(\sigma, 0,0),(1,0,1)$ or to $(\sigma, \sigma, 0)$ for some $\sigma \in \mathbb{R}$. In the case $(\sigma, 0,0)$, we have that $\varphi_{+}(\mu)=\alpha^{\sigma}(1+\mu)^{\sigma}-$ $G^{-1}-\beta$. In the case $(1,0,1)$, we have that $\varphi_{+}(\mu)=\alpha\left(1-G^{-1}\right) \mu+\alpha-\beta$. In the case $(\sigma, \sigma, 0)$, we have that $\varphi_{+}(\mu)=\left(1-G^{-1}\right) \alpha^{\sigma}(1+\mu)^{\sigma}-\beta$. In all three cases the function $\varphi_{+}$has at most a single root, which is simple. As a consequence $\varphi$ for $\alpha>0$ and $\beta \in \mathbb{R}$ has at most 4 zeroes, counted with their multiplicity.

2. Case $\alpha<0$. Consider now the case $\alpha<0$ and introduce the variable $\xi=-\alpha \mu=|\alpha| \mu$. We have that $\mu>0$ for $\xi>0$. Then $\varphi$ transforms into

$$
\varphi_{-}(\mu)=\varphi(|\alpha| \mu, \alpha, \beta)=|\alpha|^{\sigma} \mu^{\sigma}-G^{-1}|\alpha|^{\sigma_{1}+\sigma_{2}}(1+\mu)^{\sigma_{2}}-\beta .
$$


The expression for $\varphi_{-}(\mu)$ is similar as the one for $\varphi_{+}(\mu)$ in $(25)$, up to permutation of $\mu$ with $1+\mu$, and replacing $\alpha$ in $\varphi_{+}$by $|\alpha|$. Then applying to $\varphi_{-}$two steps of division-derivation procedure as we did to $\varphi_{+}$in the case $\alpha>0$, leads to the quadratic polynomial $\varphi_{-}^{2}$, defined by $\varphi_{2}^{-}(\mu)=$ $\sigma_{2}\left(\sigma-2 \sigma_{1}-1\right) \mu(1+\mu)+\sigma_{1}\left(\sigma-\sigma_{1}\right)(1+\mu)^{2}+\sigma_{2}\left(1-\sigma_{2}\right) \mu^{2}$, which is similar to (26), up the permutation of $\mu$ with $1+\mu$. Therefore, also for $\alpha<0, \beta \in \mathbb{R}$, there are at most 4 zeroes $\xi$ for $\varphi$, counted with their multiplicity.

\section{Open questions}

(1) Theorem 1 computes the cyclicity of a generic balanced canard cycle $\Gamma$ in restriction to rescaled layers. Such a rescaled layer does not define a whole neighborhood of $\Gamma$. It remains to compute the true cyclicity of $\Gamma$, i.e. to find a bound of the number of bifurcating limit cycles in a whole neighborhood of $\Gamma$. A method for achieving this result would be to blow up the system of equations (17). In such blowing up the rescaled domain may be seen as a chart of the blown-up space (the so-called family chart). To complete the study of the cyclicity it thus would remain to study the blown-up system in the other charts (the parameter charts). This does not seem to be a too difficult task.

(2) In [7], the genericity is not assumed and a result was obtained for any finite codimension for canard cycles with two breaking mechanisms (besides the two breaking parameters one considers other parameters to unfold the situation). Moreover the result was obtained in a whole neighborhood (and not just in rescaled layers), by using the Khovanskii's method directly for the non-rescaled system. The idea was to "reduce the number of exponentials". Using this procedure for canard cycles with any number of breaking mechanisms, the first step works, as it produces an equation, Det $=0$, without exponentials. Unfortunately, the number of exponentials does not decrease at the second step, as soon as there are more than 3 breaking mechanisms. It would be very interesting to find a general method to tackle the system of equations (5) in non-generic cases and for an arbitrarily number of equations.

\section{Acknowledgements}

The first author is supported by Ramon y Cajal grant RYC-2011-07730, and also partially by grants MINECO/FEDER MTM2008-03437, MINECO MTM201340998-P and AGAUR 2014SGR-568.

\section{References}

[1] P. De Maesschalk And F. Dumortier, Time analysis and entry-exit relation near planar turning point. Journal of Differential Equations, Vol. 
215 (2005), 225-267.

[2] F. Dumortier and R. Roussarie, Canard cycles and centre manifolds. Memoirs of A.M.S., vol. 121, n 577 (1996) 1-100.

[3] F. Dumortier and R. Roussarie, Canard cycles with two breaking parameters. Discrete and Continuous Dynamical Systems 17, $\mathrm{n}^{\circ} 4$, (2007), 787-806.

[4] F. Dumortier and R. Roussarie, Multiple Canard Cycles in Generalized Liénard Equations. Journal of Differential Equations 174 (2001), 1-29.

[5] F. Dumortier And R. Roussarie, Multi-layer canard cycles and translated power functions. Journal of Differential Equations 244 (2008), 13291358.

[6] A. Khovanskil, Fewnomials. Translated from the Russian by Smilka Zdravkovska. Translations of Mathematical Monographs, 88, American Mathematical Society, Providence, RI, viii + 139 (1991).

[7] L. Mahmoudi and R. Roussarie, Canard cycles of finite codimension with two breaking parameters. Qualitative Theory of Dynamical Systems 11 (2012), 167-198.

[8] D. Panazzolo, Solutions of the equation $a_{n}+\left(a_{n-1}+\ldots\left(a_{2}+\left(a_{1}+\right.\right.\right.$ $\left.\left.x^{r_{1}}\right)^{r_{2}} \ldots\right)^{r_{n}}=x$. Article in preparation (2015). 